\title{
CONCENTRACIONES DE PILAS DE ROCAS EN LA CUENCA SUPERIOR DEL RÍO SANTA CRUZ (ARGENTINA)
}

\author{
LUIS A. BORRERO*, KAREN B. BORRAZZO*, IRENE GARIBOTTI" Y MARÍA C. PALLO****
}

\section{RESUMEN}

Este trabajo presenta y caracteriza dos concentraciones de pilas de rocas identificadas en la margen sudoeste de la cuenca superior del río Santa Cruz (Argentina), en la Estancia Bon Accord. La edad mínima de estas estructuras es estimada a partir de análisis liquenométricos. Se recopila información sobre la existencia de este tipo de estructuras en la región patagónica. Por último, se discuten algunas de las funciones hipotéticas de estas acumulaciones utilizando casos arqueológicos locales y de otras partes del mundo.

PALABRAS CLAVE: pilas de rocas, liquenometría, Patagonia.

\section{CLUSTERS OF CAIRNS IN THE UPPER SANTA CRUZ RIVER BASIN (ARGENTINA)}

\begin{abstract}
Clusters of cairns located on the southwestern margin of the upper Santa Cruz river basin (Argentina), at Estancia Bon Accord are characterized in this paper. The minimum age of these structures is estimated by lichenometric analysis. Similar lithic features recorded in Patagonia are addressed and compared with those found at Bon Accord. Finally, several hypotheses about their function are evaluated using previous regional and worldwide research.
\end{abstract}

KEY WORDS: cairns, lichenometry, Patagonia.

* CONICET (IMHICIHU) y UBA. Saavedra 15 piso 5ำ (1083) Buenos Aires, Argentina, email: laborrero2003@yahoo.com, kborrazzo@yahoo.com.ar.

* IANIGLA, CCT-CONICET Mendoza, Argentina, C.C. 330, Mendoza. ireneg@mendoza-conicet.gov.ar.

** CONICET (IMHICIHU). Saavedra 15 piso 5 (1083) Buenos Aires, Argentina, email: ceciliapallo@hotmail.com. 


\section{INTRODUCCIÓN}

La búsqueda de la mayor variabilidad de situaciones arqueológicas posibles rigió el proyecto realizado durante muchos años en la cuenca superior del río Santa Cruz (Borrero 1998; Borrero y Muñoz 1999). Estos trabajos han producido un cuadro sumamente variado de hallazgos, que incluye cuevas en altura, sitios a cielo abierto estratificados o en superficie, sitios en turberas, aleros colapsados o bajo bloques, concentraciones de pinturas rupestres, etc. (Borrero y Carballo Marina 1998; Carballo Marina et al. 1999; Franco et al. 1999; García et al. 1999; Franco y Borrero 2000; Franco 2002, 2004; Belardi 2005; Borrazzo 2006, 2008; Pafundi 2006; Carballo Marina 2007; Borrero et al. 2006, 2007, 2008). A esta diversidad de situaciones arqueológicas se ha agregado el hallazgo de dos concentraciones de pilas de rocas en la margen sur del lago Argentino, a escasos kilómetros de la naciente del río Santa Cruz (Argentina) (Fig. 1). El hallazgo y estudio de estas concentraciones fue el resultado de información brindada por el Sr. Gerardo Povazsán en el marco de una búsqueda orientada de enterratorios humanos (Guichón et al. 2001) que nos llevó a evaluar una variedad de situaciones que podían aproximarse a chenques. Algunos aspectos de esta investigación forman parte de un programa de análisis de pseudoartefactos (ver Borrazzo 2011). En este caso se evalúan las condiciones ambientales bajo las cuales se pueden producir pseudoestructuras. El relevamiento arqueológico de ambos loci ha aportado escasas evidencias arqueológicas asociadas, situación que complejiza las discusiones sobre su función.

Las ocupaciones humanas en la cuenca del río Santa Cruz se remontan a comienzos del Holoceno, como lo indican los fechados radiocarbónicos disponibles para el sitio Chorrillo Malo 2 (Franco y Borrero 2003), ubicado hacia el oeste de las concentraciones, en la margen sur del lago Roca (Franco 2002). Sin embargo, las ocupaciones de la cuenca tienen una fuerte firma tardía, predominando los contextos arqueológicos fechados entre 3.300 y 170 años ${ }^{14} \mathrm{C}$ AP (Borrero y Franco 2000). Campo del Lago 2, yacimiento arqueológico localizado a unos $30 \mathrm{~m}$ del extremo oriental del lago Argentino y a menos de $10 \mathrm{~km}$ de las pilas líticas aquí estudiadas, ha sido fechado en $2.940 \pm 90$ años ${ }^{14} \mathrm{C} A P$ y, hacia el oeste, en Punta Bonita 2 se obtuvo una fecha de
$2.540 \pm 70$ años ${ }^{14} \mathrm{C}$ AP (Carballo Marina et al. 1999). Se han registrado ocupaciones más recientes tan sólo en la meseta al norte del Lago, entre 1.640 y 520 años ${ }^{14} \mathrm{C}$ AP, y al sur del lago Roca, con $170 \pm 30$ años ${ }^{14} \mathrm{C}$ AP (Carballo Marina et al. 1999; Borrero y Franco 2000). En todos los casos se trata de la utilización de nuevos sitios, en un patrón que se ha evaluado como de repoblamiento de la cuenca (Borrero y Franco 2000). Recientemente, Franco y colaboradores (2011) han obtenido la primera evidencia de ocupaciones humanas en la región en tiempos de la Anomalía Climática Medieval.

El objetivo de este trabajo es caracterizar detalladamente estas inusuales estructuras arqueológicas y enmarcarlas en antecedentes similares registrados en la región patagónica. Se informa la edad mínima de los cairns de Bon Accord que ha sido estimada mediante la aplicación de la técnica denominada liquenometría (Innes 1985). Por último, se discuten algunas de las funciones hipotéticas de estas acumulaciones utilizando casos arqueológicos locales y de otras partes del mundo.

\section{LAS PILAS DE ROCAS DE LA CUENCA SUPERIOR DEL RÍO SANTA CRUZ}

Los dos loci arqueológicos estudiados se ubican a unos 2.2 y $4.8 \mathrm{~km}$ al sur de la margen meridional del lago Argentino, en los campos de la estancia Bon Accord (Fig. 1). Los mismos presentan dos ("pilas aisladas", en adelante locus PA) y 19 (concentración de pilas, en adelante locus $\mathrm{CP}$ ) pilas de rodados. Los nódulos empleados están disponibles en el sustrato local inmediato (drift). Los loci se emplazan en espacios elevados, aunque no son los lugares más altos del paisaje. Topográficamente, $\mathrm{PA}$ y CP son de fácil acceso. Ambas lomadas son bajas y de pendiente suave. En una escala amplia, PA y CP integran el camino más corto hacia la margen sur del lago Argentino, alcanzando una pequeña bahía (Fig. 1). PA se localiza aproximadamente en la mitad del recorrido a realizar desde CP.

Las tareas llevadas a cabo para realizar el relevamiento de los loci incluyeron georreferenciamiento, registro de dimensiones, ubicación relativa, registro fotográfico de las estructuras, identificación de litologías empleadas y sus fuentes potenciales, intervenciones estratigráficas, identificación de especies vegetales presentes y estudios liquenomé- 


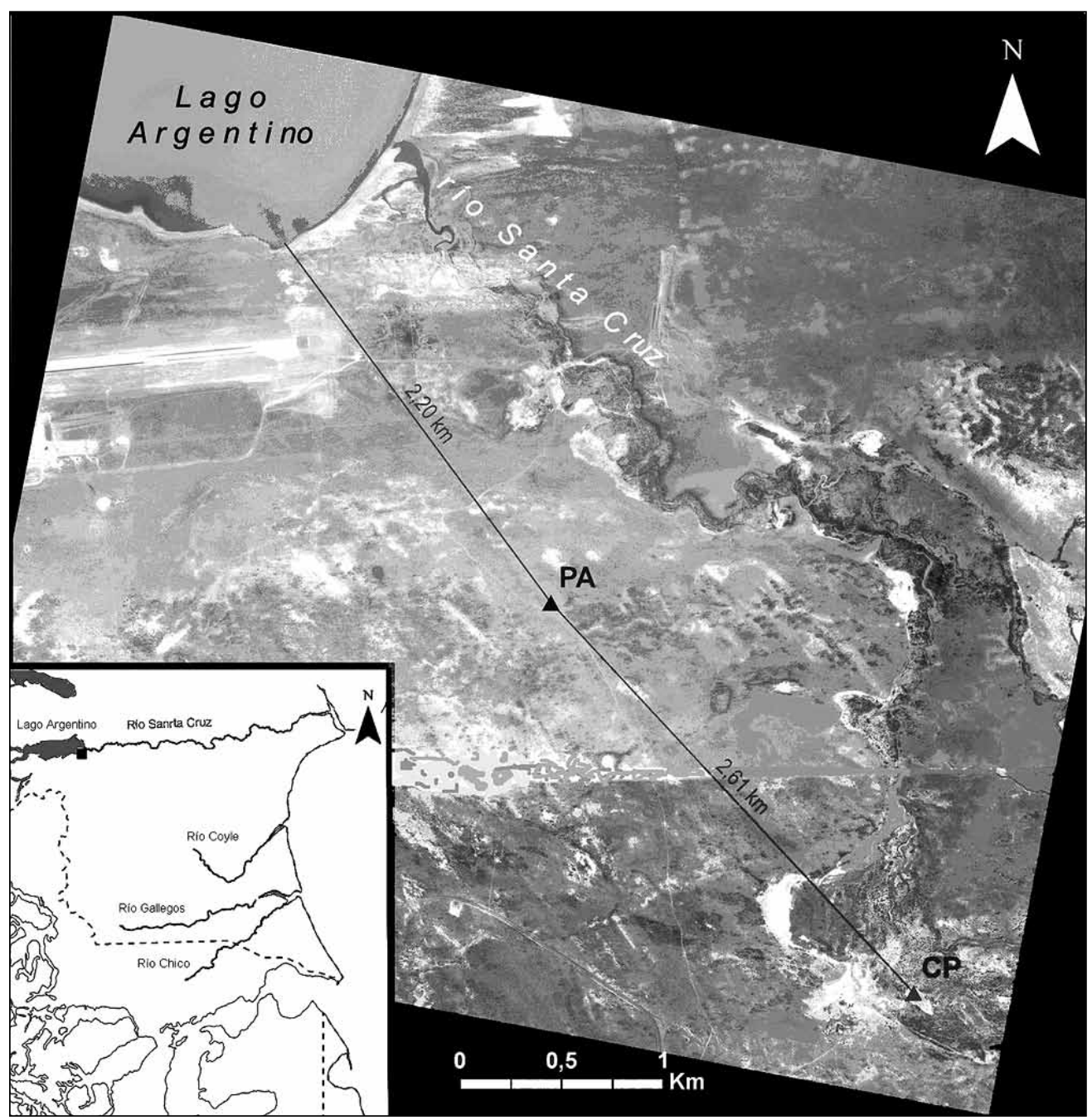

Fig. 1. Localización del área de estudio. Pilas aisladas (PA); Concentración de pilas (CP).

tricos. Las prospecciones de los loci y sus sectores adyacentes entregaron escasos artefactos líticos (ver infra). Otros registros conocidos se ubican en un peladal localizado a unos $300 \mathrm{~m}$ hacia el oeste de CP. Allí se observaron una raedera y unas pocas lascas de basalto.

\section{Descripción del locus PA}

El locus PA $\left(50^{\circ} 17^{\prime} 26.5^{\prime \prime} \mathrm{S}, 72^{\circ} 1^{\prime} 03.6^{\prime \prime} \mathrm{O}\right)$ se ubica en el flanco occidental de una lomada baja (217 $\mathrm{msnm})$. Este emplazamiento condiciona la visibilidad de las estructuras, las que sólo son visibles desde el norte y el oeste, mientras que desde el sitio la mayor visibilidad se obtiene hacia el sureste y el este.
PA presenta dos pilas de rocas cuyas dimensiones son 2.4 por $1.1 \mathrm{~m}$ (Pila 1 ) y 1.7 por $1.7 \mathrm{~m}$ (Pila 2$)$ ancho y largo, respectivamente (Fig. 2). La altura de las mismas no supera los $45 \mathrm{~cm}$ desde la superficie del terreno. El nódulo de mayor tamaño incluido en las estructuras de PA tiene 74 por 38 por $30 \mathrm{~cm} \mathrm{y}$ pertenece a la pila 1 . Las litologías observadas en las pilas no difieren de aquellas disponibles en el sustrato inmediato (Fig. 2). Estas incluyen diversas rocas metamórficas, volcánicas y sedimentarias de variada calidad para la talla. En términos de su visibilidad, PA es visible desde el O-NO; por el contrario, es invisible desde el este y/o sur puesto que las estructuras no pueden ser vistas hasta que se llega a ellas. 


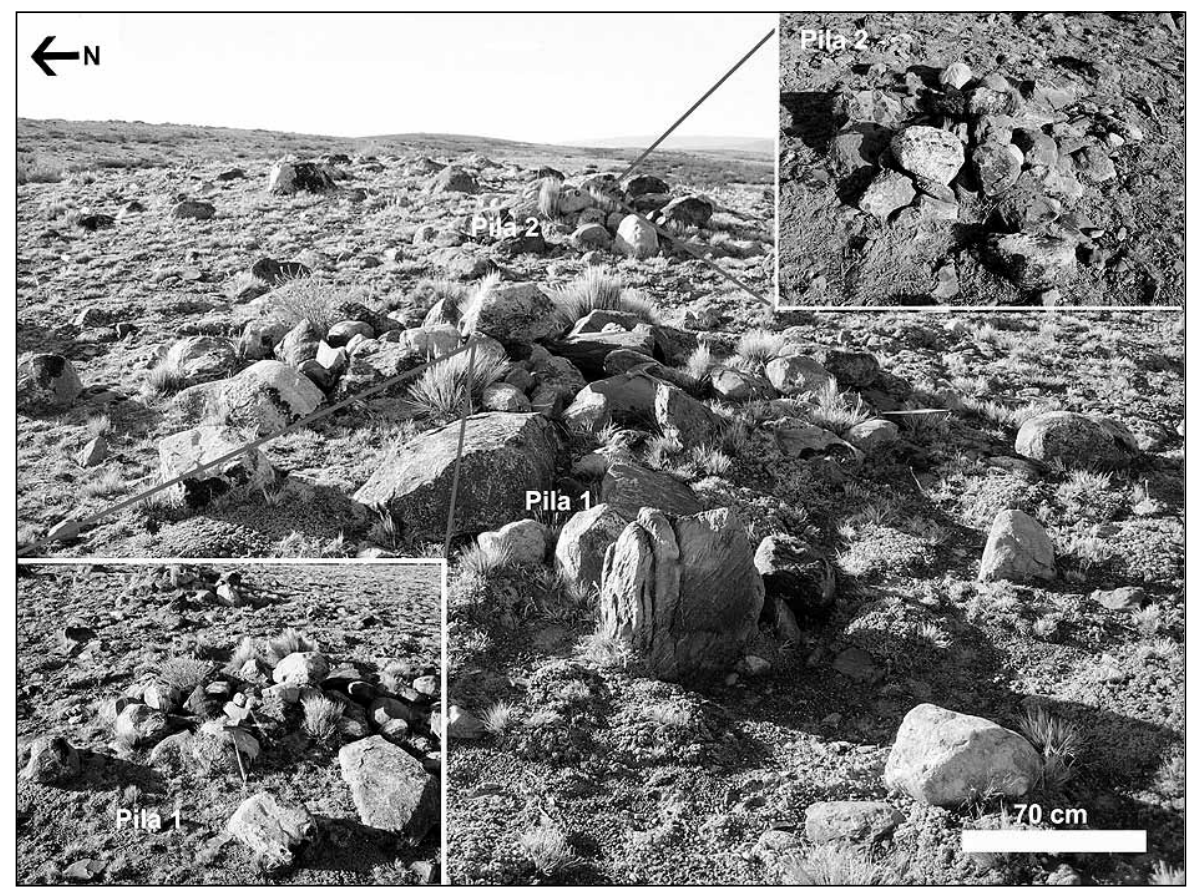

Fig. 2. Locus PA. Fotografía tomada desde el oeste.

La pila 2 se encuentra a unos $4 \mathrm{~m}$ al oeste del sector más alto de la lomada. La pila 1 se localiza a $1.76 \mathrm{~m}$ hacia el oeste de la otra estructura, y se ubica más abajo en la pendiente occidental de la pequeña elevación. Los nódulos alejados del centro de la pila 1 están dispersos, situación que contrasta con la compacidad de la pila 2 (Fig. 2). En el centro de la pila 1 se registró la presencia de una depresión de al menos $20 \mathrm{~cm}$ de profundidad desde la superficie actual. La misma se encuentra ocupada y cubierta por rodados. En la pila 2, en cambio, las rocas apoyan directamente sobre la morena y no se registró allí depresión alguna en el terreno. En ambas estructuras se observa el crecimiento de coirón entre las rocas.

En el año 2000 se sondeó una superficie de 50 por $50 \mathrm{~cm}$ en la pila 1 . Los sedimentos de los primeros $5 \mathrm{~cm}$ no estaban consolidados y presentaban color castaño claro. Inmediatamente debajo se observaron algunas rocas planas (tipo lajas) semienterradas, dispuestas horizontalmente y apoyando directamente sobre los sedimentos del depósito de drift. Estos últimos estaban muy compactados, casi impenetrables. Se alcanzaron los 50 $\mathrm{cm}$ de profundidad. La excavación sólo entregó dos mandíbulas de lagartija.

\section{Descripción del locus CP}

Unos $2.6 \mathrm{~km}$ hacia el sudeste de PA se localiza el locus CP (Fig. 1). El mismo se emplaza sobre una pequeña lomada, unos $2 \mathrm{~m}$ sobre el nivel de base del gran bajo (208 msnm) que se abre al norte del casco de la estancia Bon Accord (Fig. 3). En este locus se identificaron 19 pilas que se concentran en el extremo occidental de la loma (Fig. 4 y 5). Sus dimensiones son similares a las de las pilas de PA. $\mathrm{Al}$ igual que en ese locus, el sustrato en el entorno de CP es un depósito de drift que habría provisto la materia prima lítica para la construcción de las pilas. En el extremo oriental de la loma se observaron en superficie rodados con tamaños y litologías similares a los de las estructuras, pero su disposición espacial es dispersa. Esto constituye un elemento que refuerza el origen antrópico de las pilas de rocas de CP. Las pilas de CP conforman en planta aproximadamente un semicírculo que se cierra hacia el este y al cual se enfrenta una pila aislada (Fig. 5). El largo y ancho de la distribución es 31 y 22 m, respectivamente (Fig. 5). El perímetro de la concentración es de unos $90 \mathrm{~m}$.

$\mathrm{CP}$ es un locus altamente obstrusivo: desde la terraza localizada al sur (sobre la que se emplaza el casco de la estancia Bon Accord) la 


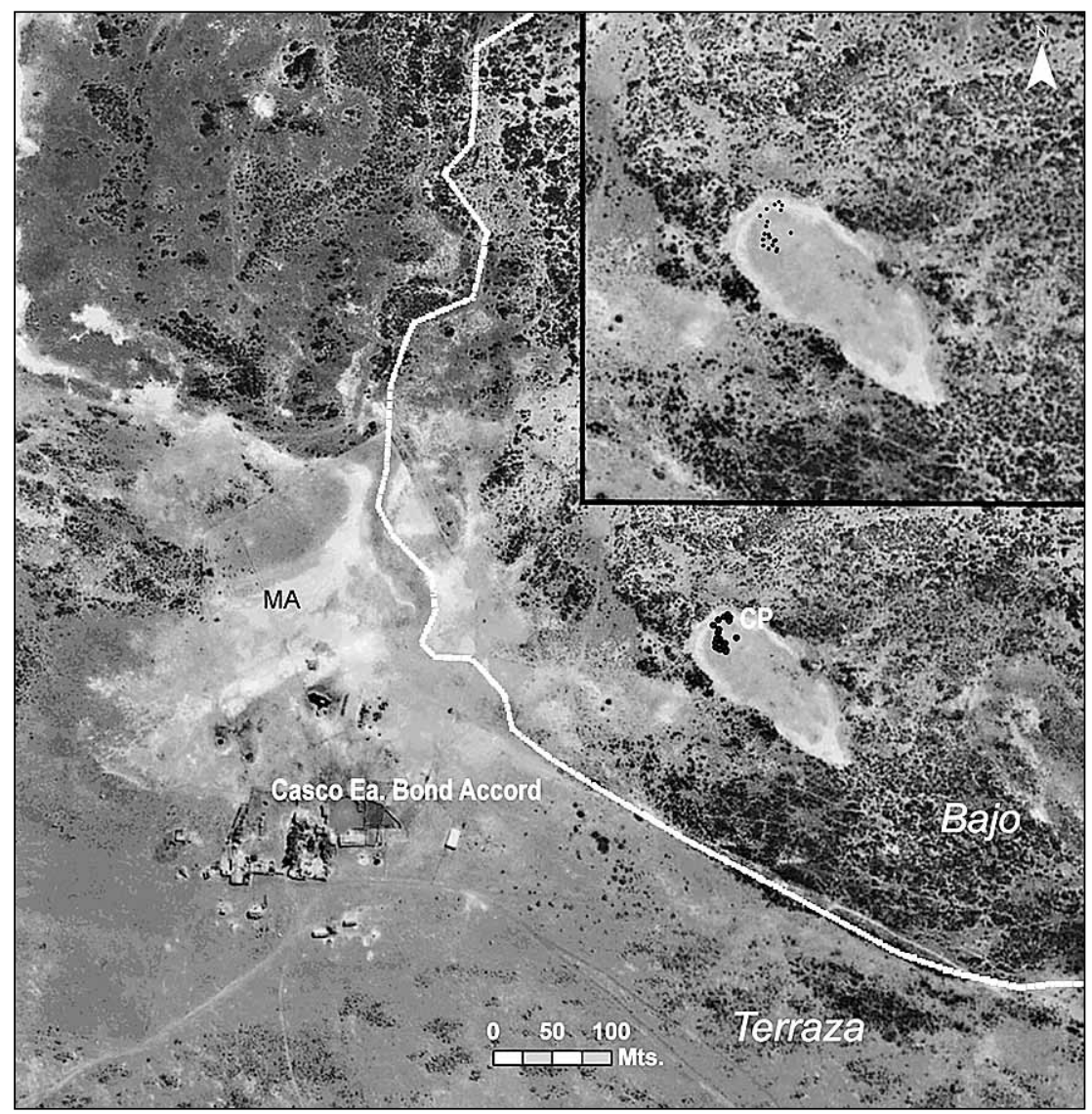

Fig. 3. Emplazamiento del locus CP en una lomada en el bajo del terreno. La línea marca el filo de la terraza (218 msnm) y el inicio del bajo. MA indica la localización de las evidencias arqueológicas más cercanas a CP. Obsérvese la cobertura vegetal diferencial entre la lomada en la que se encuentra el locus CP y el bajo.

cobertura vegetal diferencial de la loma -y no tanto su altura-constituye un factor que aumenta su visibilidad (Fig. 3). Esto marca una diferencia con PA, cuya obstrusividad es menor y su visibilidad más reducida. La cobertura vegetal de la loma es reducida (champa y coirón), siendo nula en la depresión inundable que a modo de anillo rodea el contorno de la loma (Fig. 3). El acceso a CP es sencillo; sólo el anegamiento de la depresión que rodea el locus puede haber dificultado o entorpecido el camino a las estructuras. La revisión de imágenes aéreas disponibles en el SEGEMAR (1968/69, en escala 1:10.000) permite afirmar que la cobertura vegetal diferencial de la loma de CP tiene una antigüedad mínima de 40 años. La vegetación en torno a la loma se caracteriza por la presencia de matas negras y grises (Junellia tridens y Senecio patagonicus).
La existencia de las pilas de $\mathrm{CP}$ es conocida por los habitantes de Bon Accord desde que la estancia comenzó a funcionar en 1903-1906. En la década de 1960, un empleado de la estancia excavó una de las pilas, pero no dio con huesos u otros materiales (Povazsán com. pers. 2010). El relevamiento arqueológico de $\mathrm{CP}$ entregó un pequeño conjunto de artefactos líticos $(n=7)$ localizados en el sector oriental de la lomada, varios fragmentos de gres de una botella (varios de ellos ensamblan) y un cráneo de caballo depositado sobre una de las pilas. En enero de 2011, el mismo se presentó en tres grandes fragmentos, con meteorización variable entre $3 y$ 4 , y varios fragmentos menores en estadio 5 . Los dientes estaban muy destruidos, algunos aún dentro de sus alvéolos y otros sueltos y muy fragmentados. Apoya sobre sustrato limoso a una distancia de unos $100 \mathrm{~cm}$ de la estructura 877. 


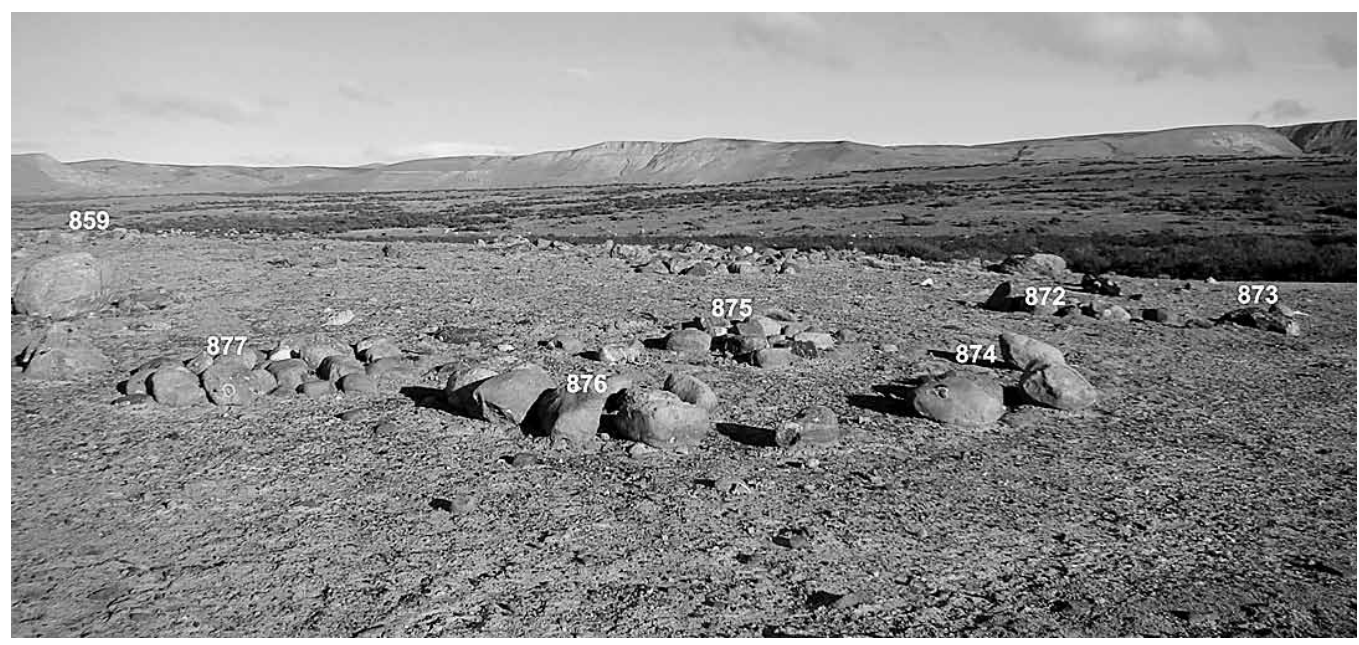

Fig. 4. Locus CP (fotografía tomada desde el oeste). Los números indican la denominación de las pilas.

En el 2000, realizamos un sondeo de 50 por $50 \mathrm{~cm}$ en la estructura 872 , cuyas dimensiones son 2.90 por $1.70 \mathrm{~m}$. El mismo alcanzó $50 \mathrm{~cm}$ de profundidad. Los sedimentos de los primeros 10 $\mathrm{cm}$ son de color gris. Por debajo, los mismos se tornan castaños claros y se agrega la presencia de bloques de diferentes tamaños. La compacidad y resistencia de este sustrato dificulta la intervención estratigráfica. Entonces, la impenetrabilidad del depósito tiene implicaciones tafonómicas, pues inhibiría la incorporación y/o migración vertical de otros materiales (i.e. huesos, artefactos).

La inspección de los nódulos que componen las estructuras mostró que algunos de ellos aún conservan el barniz de suelo que remite a posiciones previas en estado semienterrado, lo que podría sugerir que para la construcción de las pilas se removieron rodados parcialmente expuestos en el depósito subyacente.

\section{Los artefactos líticos}

Aún cuando los loci fueron visitados e inspeccionados en varias oportunidades, los artefactos recién fueron identificados en el último relevamiento realizado. Esto ha sido consecuencia de la baja obstrusividad de los mismos sobre el sustrato local (que incluye nódulos de rocas de diverso tamaño y materia prima) y al estado semienterrado de algunas de las piezas. En el último caso, si bien el proceso predominante en las geoformas donde se emplazan las pilas es la erosión, existen en escala micro pockets capaces de retener diferencialmente los sedimentos transportados haciendo posible el sepultamiento.

El conjunto lítico recuperado en PA proviene de una superficie de $50 \mathrm{~m}^{2}$ localizada en la cumbre de la lomada, al E de las pilas. Tres de los cuatro artefactos recuperados se encontraban semienterrados en el momento del hallazgo y dos de ellos exhiben crecimiento de líquenes (Rhizocarpon geminatum)

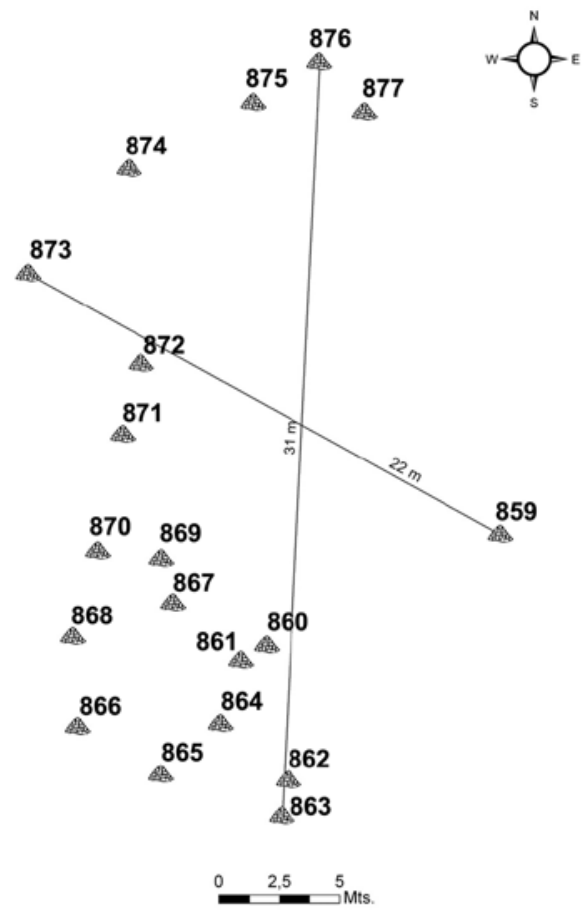

Fig. 5. Esquema de la distribución de pilas en el locus CP. 


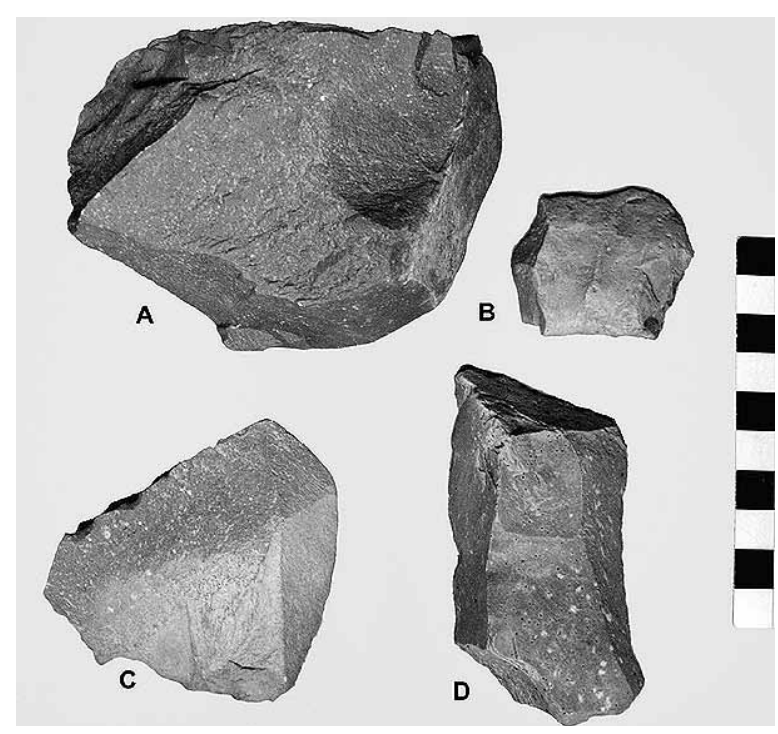

Fig. 6. Artefactos recuperados en PA.

sobre la superficie expuesta. La superficie enterrada o en contacto con el sustrato de cada pieza presenta un rock coating del tipo barniz de suelo (i.e. A en Fig. 6). Estos tres elementos sugieren la existencia de condiciones estables en el contexto posdepositacional. La muestra está integrada por tres lascas y un núcleo. Aún cuando no se registraron relaciones de ensamblaje, las características macroscópicas de la corteza y materia prima (dacita gris verdosa) de las cuatro piezas sugieren que habrían resultado de la talla de un mismo nódulo. El núcleo (A en Fig. 6) exhibe un flanco con extracciones paralelas a la vez que registra lascados multidireccionales aislados en otros sectores. Los desechos recuperados incluyen una lasca de arista (D en Fig. 6) y otra angular (C en Fig. 6), ambas coherentes con las estrategias de reducción registradas en el núcleo. El tercer desecho ( $\mathrm{B}$ en Fig. 6) es un fragmento distal de lasca con $75 \%$ de reserva de corteza. Los filos naturales de las lascas exhiben esquirlamientos irregulares y discontinuos, algunos de ellos más frescos, lo que sugiere su origen tafonómico.

La muestra artefactual recuperada en CP está compuesta por seis artefactos líticos recolectados en una superficie de $100 \mathrm{~m}^{2}$. Los instrumentos dominan la muestra $(n=6 / 7)$ y están representados por tres raederas (dos de ellas sobre lasca extraída de núcleo preparado, A y $\mathrm{B}$ en Fig. 7), un raspador con evidencias de reactivación, una lasca con rastros complementarios y un artefacto con marcas

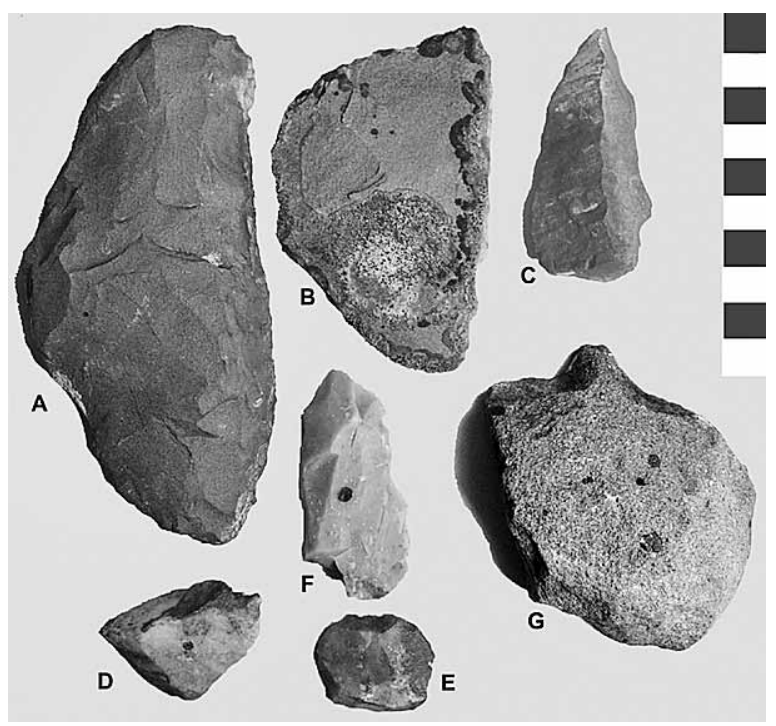

Fig. 7. Artefactos recuperados en $\mathrm{CP}$.

de percusión una punta entre muescas ( $G$ en Fig. 7). El raspador ensambla (sensu Ramos 1994) con el único desecho recuperado (un fragmento proximal de lasca angular de calcedonia coloreada con impurezas). La forma base del raspador es el fragmento distal de la lasca, la que ha sido fracturada intencionalmente por percusión sobre la cara dorsal, produciendo una fractura radial (sensu Weitzel 2010) (D y E en Fig. 7). Por lo tanto, es posible plantear que el raspador ha sido manufacturado, utilizado, reactivado y descartado en el sitio. La calcedonia de ambos artefactos es macroscópicamente similar a una de las variedades registrada en los conjuntos líticos posteriores al 1.500 AP recuperados en el sitio Cerro León 3 (periferia meridional de la sierra Baguales) (Borrazzo 2006). Por otra parte, la roca lutítica utilizada en la confección de la raedera de mayor tamaño (A en Fig. 7) es compatible con aquellas disponibles en grandes nódulos en la misma región (Borrazzo 2006, 2008). Ambos elementos sugieren conexiones a uno y otro lado de las sierras Baguales, como fuera previamente sostenido (Franco y Borrero 2000).

Sobre tres artefactos (los de mayor peso y tamaño; A, B y G en Fig. 7) se registró el crecimiento de líquenes crustosos, algunos de ellos pertenecientes a Rhizocarpon geminatum. En un caso, los mismos cubren ca. 50\% de la cara artefactual expuesta (B en Fig. 6). Este es un elemento que sugiere condiciones de estabilidad en la vida postdepositacional de esos 


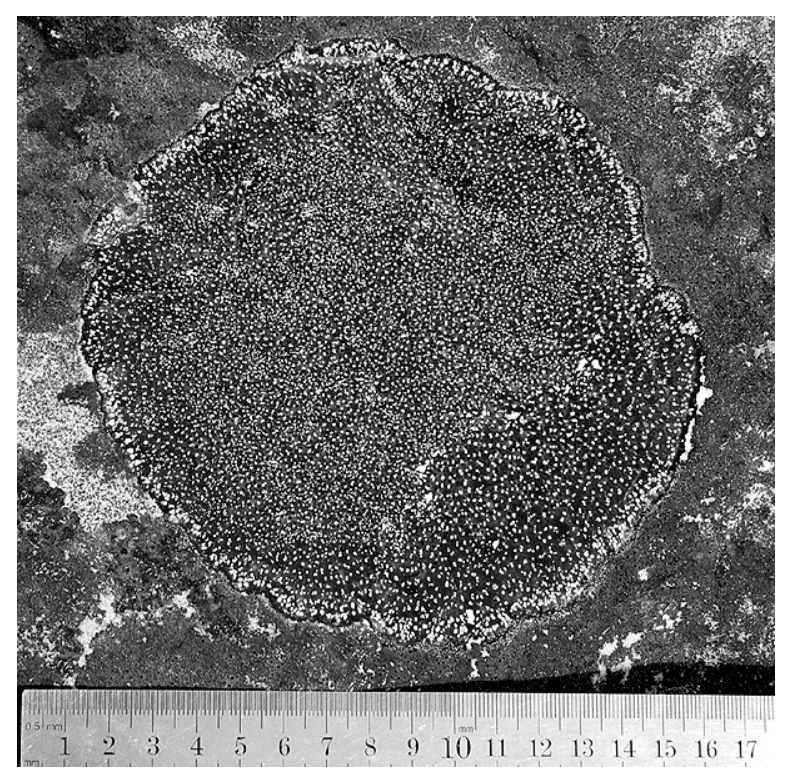

Fig. 8. Espécimen de Rhizocarpon subg. Rhizocarpon.

artefactos y que su disponibilidad en superficie no es un fenómeno reciente.

\section{Líquenes y cronología}

Los líquenes son colonizadores tempranos de superficies rocosas. Su crecimiento es muy lento y son organismos muy longevos, estimándose que algunas especies pueden vivir más de 5.000 años. El crecimiento de los líquenes es radial, siendo posible estimar la edad de un liquen a partir de su diámetro. Estas características hacen que los líquenes sean organismos especialmente útiles para datar superficies de edad desconocida, mediante la técnica conocida como liquenometría (Innes 1985).

Existen distintas aproximaciones para utilizar los líquenes para datar una superficie (ver revisión en Bradwell 2009). La aproximación tradicional consiste en medir el diámetro de los líquenes más grandes que crecen en la superficie. Conociendo la tasa de crecimiento radial del liquen es posible estimar la edad mínima de exposición de la superficie a partir del diámetro del espécimen más grande encontrado en el sitio. Para ello generalmente se utilizan especies de líquenes crustosos (líquenes que crecen estrechamente unidos al sustrato), principalmente del género Rhizocarpon subgénero Rhizocarpon (Fig. 8). La utilización de este método en arqueología es cuestionable debido a la dificultad de determinar si el liquen

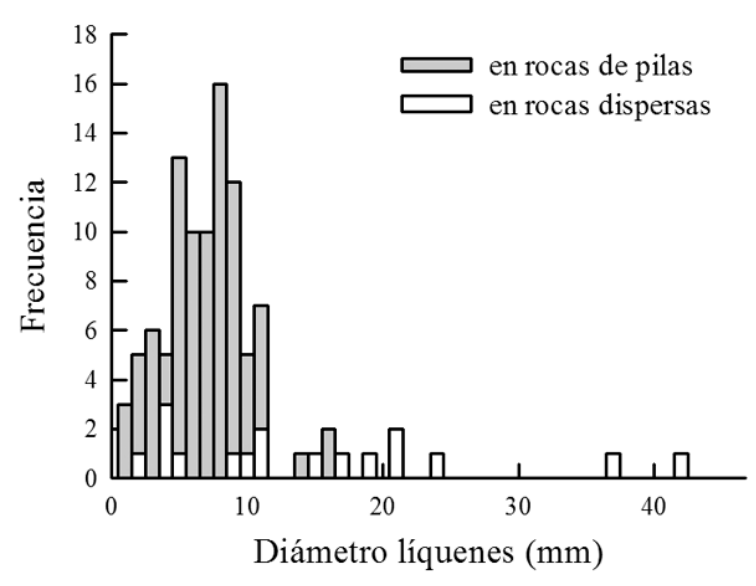

Fig. 9. Distribución de frecuencia de los diámetros de los especimenes del subgénero Rhizocarpon registrados en las rocas de las pilas y otras dispersas en los loci.

más grande encontrado se estableció posteriormente a la construcción de la estructura arqueológica, o es un individuo que se estableció previamente en la roca y sobrevivió a su traslado. Este problema puede ser abordado utilizando una aproximación liquenométrica poblacional. El análisis de las distribuciones de frecuencia de tamaños (size-frequency approach) se basa en el tamaño de la totalidad de los líquenes de la población que crece en la superficie. Mediante este método es posible identificar si la población corresponde a uno o varios eventos de colonización, y si el talo más grande encontrado en la superficie ha sido heredado o es preexistente al resto de la población (Benedict 1967; McCarroll 1994; Bull y Brandon 1998; Bradwell 2004).

Otras aproximaciones liquenométricas se basan en estudios de la composición florística y estimaciones de la cobertura total de la comunidad de líquenes. Se supone que la cobertura de una o varias especies de líquenes aumenta en forma regular con el paso del tiempo y que la composición de especies varía en función a procesos sucesionales. Estas aproximaciones permiten realizar dataciones relativas de la fecha de exposición de distintas superficies (Benedict 1967; Innes 1986).

En el presente estudio, las cuatro aproximaciones liquenométricas descriptas anteriormente fueron consideradas para datar las pilas de rocas. Para ello, en cada una de las pilas se estudiaron 
todas las rocas que las conforman o un mínimo de 15 rocas. Además, se estudiaron 100 rocas dispersas en la matriz circundante a las pilas de rocas. En cada roca se midió el diámetro mayor del espécimen más grande de Rhizocarpon subgénero Rhizocarpon, se registró la diversidad de especies y se estimó la cobertura porcentual de cada especie. Muestras de las especies de líquenes presentes fueron colectadas y llevadas de vuelta al laboratorio para corroborar su filiación taxonómica usando técnicas morfológicas, anatómicas y quimiotaxonómicas.

Se relevaron un total de 363 rocas en las pilas, en las cuales se encontraron 95 ejemplares de Rhizocarpon subgénero Rhizocarpon. La distribución de frecuencia de tamaños de los líquenes es unimodal (Fig. 9), sugiriendo que los mismos constituyen una única población coherente. Los líquenes de mayor tamaño forman parte de esta distribución, por lo que no hay evidencias para sospechar que alguno de ellos sea un individuo preexistente a la construcción de las pilas. Es más, los líquenes en las rocas de las pilas son de menor tamaño que los encontrados en las rocas del entorno (diámetro máximo 16 vs. 42 $\mathrm{mm}$ en diámetro, respectivamente), lo que sugiere que los líquenes de las pilas son más jóvenes y no han sido heredados de una población previa. En las rocas dispersas en muchos casos fue imposible medir ejemplares de $R$. subgénero Rhizocarpon ya que se encontraron mosaicos resultantes de la coalescencia de los talos, lo cual es una situación típica en poblaciones de gran edad. Finalmente, los gráficos de distribución de frecuencia de tamaños de los líquenes más grandes muestran una clara diferenciación entre las poblaciones de líquenes en las rocas de las pilas $y$ en las rocas dispersas en el entorno, sugiriendo que corresponden a distintas poblaciones (Fig. 9). Todos estos indicios validan el uso de los ejemplares de $R$. subgénero Rhizocarpon para estimar la edad de construcción de las pilas de rocas.

Para realizar la datación cuantitativa de las pilas de rocas, el tamaño de los especímenes de $R$. subgénero Rhizocarpon debe ser relacionado a una tasa de crecimiento para la especie en el ambiente de estudio. Para ello, en el presente trabajo se registraron datos del diámetro de los líquenes de este subgénero en seis sitios de referencia localizados en la estepa patagónica, bajo condiciones ambientales que se asumen similares a las del sitio de estudio. Los sitios de referencia incluyen estructuras histó- ricas y lápidas funerarias cuya fecha de exposición pudo acotarse con precisión. Los sitios de referencia tienen una antigüedad de 89 a 114 años. A partir de estos datos, la tasa de crecimiento del subgénero Rhizocarpon en la estepa patagónica se estima en el rango entre 0.061 y $0.184 \mathrm{~mm}$ año ${ }^{-1}$. Es importante destacar que el cementerio de la localidad de El Calafate, localizado a $10 \mathrm{~km}$ del sitio de estudio, fue relevado sin éxito en busca de ejemplares de $R$. subgénero Rhizocarpon. La lápida más antigua en este cementerio databa de principios del siglo XX. Esto sugiere que el ambiente local de estudio es adverso al establecimiento $\mathrm{y} / \mathrm{o}$ crecimiento de estos líquenes, posiblemente debido a la proximidad de la cordillera de los Andes, desde donde llegan vientos fríos y secos. Por lo tanto, nosotros asumimos que la tasa de crecimiento más baja estimada en los sitios de referencia es la mejor aproximación a la tasa de crecimiento en la zona de estudio. Aplicando esta tasa de crecimiento $\left(0.061 \mathrm{~mm}^{2} \mathrm{no}^{-1}\right)$ al tamaño del talo más grande encontrado ( $16 \mathrm{~mm}$ ), se estima que la fecha mínima de construcción de las pilas de rocas bajo estudio es de 260 años.

El análisis de la composición florística y cobertura de las comunidades epilíticas en las rocas estudiadas muestra la presencia de 21 especies de líquenes, una especie de algas y una especie de musgos. La cobertura total es mayor en las rocas dispersas que en las rocas que conforman las pilas (76.1 y $47.9 \%$, respectivamente). Las comunidades en las rocas de las pilas y en las rocas dispersas presentan una gran similitud en su composición florística, pero diferencias importantes en la cobertura relativa de las especies. En las rocas dispersas la comunidad está dominada por Rhizocarpon geminatum, que en promedio cubre $40 \%$ de la superficie de las rocas. En cambio, en las rocas de las pilas $R$. geminatum cubre solamente el $16 \%$ de la superficie de las rocas, mientras que otras especies tales como Xanthoparmelia squamans, Rhizoplaca melanophthalma y Xanthoparmelia mougeotii también presentan alta cobertura $(5.2,6.3$ y $8.6 \%$ de cobertura en las rocas, respectivamente). Estos datos sugieren que en la medida que la sucesión ecológica avanza, $R$. geminatum gana dominancia en las comunidades, reemplazando a otras especies de líquenes. Esto coincide con el patrón de reemplazo de especies observado en el proceso de sucesión primaria en ambientes de montaña próximos al área 
de estudio del presente trabajo (Garibotti et al. 2011). Por lo tanto, los resultados sugieren que el análisis de la composición florística y/o la estimación de la cobertura total de las comunidades de líquenes es una herramienta útil para estimar la edad relativa de distintas superficies en la estepa patagónica. Asimismo, en el caso de estructuras cuyo origen antropogénico sea cuestionable, la cobertura y composición florística de la comunidad de líquenes saxícolas sería una evidencia adicional a favor o en contra de la hipótesis.

\section{LAS PILAS DE BON ACCORD EN EL CONTEXTO REGIONAL}

La presencia de pilas conformadas por rocas disponibles en el sustrato inmediato hace indispensable la evaluación de la hipótesis que postula la génesis natural de esas estructuras (Tilley et al. 2000). Esto implica identificar los procesos geológicos y geomorfológicos disponibles localmente en el pasado y el presente para establecer si los mismos pueden ser responsables de los patrones materiales observados (Schiffer 1987; Waters 1996). La revisión bibliográfica realizada sugiere que nada semejante ha sido registrado en la literatura específica (Wood y Johnson 1978; Waters 1996), o que pueda derivar de las condiciones geológicas de la región (Haller 2002). Nuestras observaciones en Patagonia nos permitieron identificar algunas condiciones bajo las cuales se crean acumulaciones de rocas. Por ejemplo, en la estancia La Verdadera Argentina se registró lo que denominamos Fábrica Tafonómica, que es una concentración de grandes "lascas" producidas por los impactos generados durante la caída de bloques en el talud del cerro Tridente (Borrazzo 2008). Situaciones similares han sido observadas en Pali Aike (Santa Cruz) o en otras regiones, como los Castillos de Pincheira (Mendoza) (obs. pers. de los autores 1980, 2006). Sin embargo, los contextos geomorfológicos y los patrones espaciales que exhiben las acumulaciones naturales en los casos mencionados difieren marcadamente de las estructuras de Bon Accord.

Concentrándonos en los casos de estructuras de rocas, son muy variados los ejemplos registrados en la arqueología de Patagonia meridional, la mayoría de las cuales tienen una atribución funcional relativamente bien consensuada (Martinic 2002). Los más frecuentemente mencionados en la bibliografía regional son los chenques. En general, se trata de estructuras de piedras acumuladas de contorno oval, elíptico o circular, de unos 3 a $5 \mathrm{~m}$ de largo por otros tantos de ancho ... altura máxima de las acumulaciones ... $50 \mathrm{~cm}$ (Goñi y Barrientos 2000: 168; Reyes 2002: 94) construidas con fines funerarios. Sin embargo, también los hay más pequeños (Goñi y Barrientos 2000: 166-167). La profundidad de los entierros se limita a unos pocos centímetros por debajo de las piedras que cubren los cuerpos (Reyes 2002). Los chenques del lago Salitroso fueron construidos con rocas localmente disponibles y se cuentan casos de chenques sin entierros (en el NW de Santa Cruz, Goñi y Barrientos 2000: 169, Goñi et al. 2004: 1104). Se ha hablado de pseudochenques o chenques "falsos" en el caso de Río Cisnes, Aisén (Reyes et al. 2006: 80). Se trata de acumulaciones intencionales de piedras que incluyen "acumulaciones vacías", sin ningún elemento cultural, bioantropológico y/o ecofactual" (Reyes et al. 2006: 82). Los autores destacan que aún en la sección media del valle del río, donde son escasas las elevaciones, ocho de las trece estructuras identificadas se ubican en cimas de cerros de mínimo relieve y tres lo hacen en laderas (Reyes et al. 2006: 84). Los pseudochenques se han considerado marcadores territoriales (Goñi y Barrientos 2000, Reyes et al. 2006) o tumbas prepreparadas (Goñi et al. 2004).

También se cuenta con casos en los que la estructura del chenque está desarmada y la ausencia de huesos es fácilmente interpretable como el producto de la meteorización (Zubimendi 2010: 512; Zilio y Zubimendi 2011). En la cumbre del cerro Convento (Santa Cruz) hemos registrado un chenque desarmado en cuya superficie se observó un hueso humano en avanzado estado de meteorización (obs. pers. de los autores 2006).

Por otra parte, en Comallo (Río Negro) se ha registrado la existencia de una estructura circular de ca. $16 \mathrm{~m}$ de diámetro formada por rocas de aproximadamente $20 \mathrm{~cm}$ disponibles localmente. La misma se localiza sobre una lomada baja. No se apreciaron materiales arqueológicos asociados (Eduardo Crivelli com. pers. 2011).

Otro tipo de estructura construida por cazadores-recolectores en la región a partir de la acumulación de rocas son los parapetos, identificados en distintas mesetas patagónicas (Gradín 1962, 1971, 
1976). Se trata de reparos, de forma semicircular, cuyas dimensiones son 50-70 m de alto y unos 5 $\mathrm{m}$ de diámetro, que suelen presentarse agrupados $y$ han sido interpretados como bases para toldos, apostaderos de caza y/o loci de procesamiento (Aragone y Cassiodoro 2005-2006, Goñi 2010).

Ya para tiempos históricos, se registra el uso de acumulaciones de rocas con diferentes propósitos. Este es el caso de los cairns producidos por varias de las expediciones de exploración en los siglos recientes. Ejemplo de ello lo es el cairn realizado por Agassiz en Monte Aymond. Por su parte, Martinic y Porter (2001: 91-92) presentan una fotografía de un cairn histórico que ha sido removido y mencionan el caso de Parker King, quien recuperó el testimonio que Antonio de Córdova había dejado en un cairn en 1789 (Martinic y Porter 2001: 101).

Otra función cumplida por pilas de rocas en tiempos históricos es para postación de alambrados (Martinic 2002:104-105). En este caso, se trata de conjuntos de pilas que presentan una disposición lineal.

Recientemente se ha comenzado a explorar el rol de estructuras de rocas construidas por cazadores-recolectores como marcadores astronómicos. Este es el caso de los estudios realizados por Romiti (2010) en el sitio cementerio Chenque 1 (La Pampa, Argentina). Los resultados obtenidos en su trabajo sugieren el uso de acumulaciones antrópicas de rocas alineadas con fenómenos astronómicos para la segmentación del ciclo anual y el desarrollo de actividades rituales en grupos de cazadoresrecolectores del Holoceno tardío.

\section{DISCUSIÓN Y CONCLUSIONES}

La información disponible permite sostener que las pilas de rocas que integran $\mathrm{CP}$ y $\mathrm{PA}$ son acumulaciones artificiales. Su morfología sugiere que no se trataría de estructuras habitacionales históricas o reparos, como los parapetos identificados en las mesetas patagónicas (Gradín 1962, 1971, 1976) y su disposición espacial sugiere que no se relacionan con alambrados (Martinic 2002). Las rocas incluidas en CP y PA no presentan alteraciones térmicas, por lo que no se habrían usado para fuegos (a la manera de "marcadores discontinuos").

Las acumulaciones de rocas indican la intencionalidad de señalar o implicar "algo". Una explicación posible es que estas estructuras hayan sido utilizadas como depósitos de carne, como los excavados por Frison (Frison y Todd 1986), o los descriptos por Binford entre los Nunamiut: The cache at the camp consisted of a small circle of stream boulders weighing between 12 and $20 \mathrm{lb}$; somewhat smaller stones were placed in the center to raise the meat off the ground (Binford 1978: 291, ver también p. 461). El hallazgo realizado por Bird de una acumulación de rocas cubriendo los restos de un perezoso en la cueva Pali Aike (Bird 1988: 108) puede ser interpretado de esta manera, o como una variante de cubrir con rocas materiales ofensivos o molestos para la ocupación humana (Martin 2009).

En otras oportunidades se han registrado concentraciones de pilas de rocas con disposición circular o semicircular asociadas a actividades rituales, como es el caso del sitio de sacrificio y ofrendas Saami en Grythaugen (Noruega) (Bradley 2000:10). Una característica destacable de este tipo de sitios es la baja diversidad de evidencias asociadas a las estructuras, lo que destaca la especificidad funcional de ese espacio (Bradley 2000). Asimismo, se debe considerar la alternativa de que algunas de estas estructuras hayan funcionado como marcadores astronómicos. Los numerosos registros de Canadá han sido considerados landmarks o marcadores territoriales (Aporta 2009), puntos de observación, trampas para zorros u otros animales, o lugares asociados con muertes (Hallendy 2009).

Una alternativa apropiada para discutir PA es que se trate de cairns históricos, como aquellos relacionados con las expediciones de exploración en los siglos recientes, alternativa que la cronología disponible no avala.

Hay que recordar que existen otras acumulaciones de rocas similares en la región cuya función permanece desconocida. Tal es el caso de las estructuras circulares de las cuencas del Centinela Chico y Centinela, y otras identificada recientemente al este de las aquí presentadas (Franco et al. 1999; Franco com. pers. 2011). Esto complica no sólo el tema de la contemporaneidad de las estructuras que, a partir de algún alineamiento se pudieran considerar, sino también que invita tanto a realizar una prospección mucho más intensa y completa orientada específicamente hacia el reconocimiento de este tipo de acumulaciones, como a integrar resultados obtenidos por otros equipos antes de desarrollar esta discusión. 
En suma, hemos presentado información relacionada con estructuras de funcionalidad desconocida, cuya construcción podemos remitir a un período anterior a la exploración europea y colonización ganadera del interior de la Patagonia meridional. Estas estructuras forman parte de la señal arqueológica generada por sociedades de cazadores-recolectores que utilizaban la cuenca superior del río Santa Cruz a fines del Holoceno, momento para el que no es abundante la evidencia de presencia humana.

Presentamos nuestros datos como resultado de conexiones posibles entre las localizaciones que conocemos. Hemos evaluado alternativas funcionales para las estructuras de Bon Accord. Si bien hemos podido descartar algunas de ellas, son varias las que aún permanecen en pie. Pero entendemos que esto no debe ser un impedimento para comunicar el caso a la comunidad arqueológica. Como mínimo agrega casuística y variación a la tipología de estructuras de piedra de Martinic, variación al registro arqueológico y preguntas a la investigación planteada.

\section{AGRADECIMIENTOS}

Agradecemos al Sr. Gerardo Povaszán por la información brindada y por su apoyo permanente a los trabajos del Proyecto Magallania. A Cristian Favier Dubois, Ricardo Guichón, Fabiana Martin, Nora Franco y Ramiro Barberena por su participación en los trabajos de campo, y a Marcelo Vittores su información. Esta investigación se llevó a cabo en el marco de los proyectos "Circulación humana en el extremo sur de la Patagonia" y "Sitios anómalos, distribución de poblaciones arqueológicas y redes regionales de interacción", financiados por la Universidad de Buenos Aires, y del proyecto CRN II \# 2047, financiado por el Instituto Interamericano para Investigación en Cambio Global (IAI).

\section{BIBLIOGRAFÍA}

APORTA, C. 2009. The Trail as Home: Inuit and Their PanArctic Network of Routes. Human Ecology, 37:131-146.

ARAGONE, A. y G. CASSIODORO 2005-2006. Los parapetos de Cerro Pampa: registro arqueofaunístico y tecnológico (noroeste de la provincia de Santa Cruz). Arqueología, 13:131-154.
BELARDI, J.B. 2005. Paisajes Arqueológicos: un estudio comparativo de diferentes ambientes patagónicos. Archaeopress, Oxford.

BENEDICT, J.B. 1967. Recent glacial history of an alpine area in the Colorado Front Range, U.S.A. 1. Establishing a lichen-growth curve. Journal of Glaciology, 6:817-832.

BINFORD, L.R. 1978. Nunamiut etnoarchaeology. Nueva York, Academic Press.

BIRD, J. 1988. Travels and Archaeology in South Chile, editado por J. Hyslop. University of lowa Press, lowa.

BORRAZZO, K. 2006. Tecnología lítica del alero Cerro León 3 (Santa Cruz, Argentina). Magallania, 34(2):63-74.

2008. Distribuciones artefactuales en la periferia sudeste de la sierra Baguales (Santa Cruz, Argentina). Magallania 36(1):103-116.

2011. Tafonomía lítica y pseudoartefactos: el caso de la península El Páramo (Tierra del Fuego, Argentina). Intersecciones en antropología 12:261-273.

BORRERO, L.A. 1998. Arqueología de la Patagonia Meridional (Proyecto Magallania). Ediciones Búsqueda de Ayllu, Concepción del Uruguay.

2007. Longitudinal Taphonomic Studies in Tierra del Fuego, Argentina. En: Taphonomy and Zooarchaeology in Argentina, editado por Gutierrez M., L. Miotti, G. Barrientos, G. Mengoni Goñalons y M. Salemme, pp. 219-233. BAR Internacional Series 1601, Oxford.

BORRERO, L., R. BARBERENA, F. MARTIN y K. BORRAZZO 2007. Collapsed Rockshelters in Patagonia. En: On Shelter's Ledge: Histories, Theories, and Methods of Rockshelter Research, editado por M. Kornfeld, Sergey Vasil'ev y Laura Miotti, pp. 135-139. BAR International Series, Oxford.

BORRERO, L.A. y F. CARBALLO MARINA 1998. Proyecto Magallania: La cuenca superior del tío Santa Cruz. En: Arqueología de la Patagonia Meridional (Proyecto Magallania), editado por Borrero L.A., pp. 11-27. Ediciones Búsqueda de Ayllu, Concepción del Uruguay. BORRERO L.A. y A.S. MUÑOZ 1999. Tafonomía en el bosque patagónico. Implicaciones para el estudio de su explotación y uso por poblaciones humanas de cazadoresrecolectores. En: Soplando en el viento, pp. 43-56. Universidad Nacional del Comahue, Neuquén.

BORRERO, L.A., J. CHARLIN, R. BARBERENA, F.M. MARTIN, K. BORRAZZO y G.L. L'HEUREUX 2008. Circulación humana y modos de circulación al sur del río Santa Cruz. En: Arqueología del extremo sur del continente americano. Resultados de nuevos proyectos, compilado por Borrero L.A. y N. Franco, pp. 155-174. CONICET-IMHICIHU, Buenos Aires. 
BORRERO, L.A. y N.V. FRANCO 2000. Estrategias de Utilización de Sierra Baguales. Actas del XIV Congreso de Arqueología Chilena, pp: 269-283. Copiapó.

BORRERO, L.A., N.V. FRANCO, F.M. MARTIN, R. BARBERENA, R. GUICHON, J.B. BELARDI, C. FAVIER DUBOIS y L. L'HEUREUX 2006. Las Cabeceras del Coyle: información arqueológica y circulación de poblaciones humanas. En: Pasado y Presente en la cuenca del río Coyle, editado por Carballo Marina, F., J. Belardi y S. Espinosa, pp. 75-95. Universidad Nacional de la Patagonia Austral, Unidad Académica de Río Gallegos. Río Gallegos.

BRADLEY, R. 2000. An archaeology of natural places. Routledge, Londres.

BRADWELL, T. 2004. Lichenometric dating in Southeast Iceland: the size-frequency approach. Geografiska Annaler, 86:31-41.

BRADWELL, T. 2009. Lichenometric dating: A commentary, in the light of some recent statistical studies. Geografiska Annaler, 91:61-69.

BULL, W.B. y M.T. BRANDON 1998: Lichen dating earthquakegenerated regional rockfall events, Southern Alps, New Zealand. Geological Society of America Bulletin, 110:60-84.

CARBALLO MARINA, F. 2007. La cuenca superior del río Santa Cruz: las poblaciones humanas y el uso del espacio. Tesis doctoral inédita. Universidad Nacional de La Plata, La Plata. MS.

CARBALLO MARINA, F., L.A. BORRERO, N.FRANCO, J.BELARDI, V. HORWITZ, A. MUÑOZ, P. CAMPAN, F. MARTIN, F. BORELLA, M. GARCÍA, F. MUÑOZ, F. SAVANTI y J.LANATA 1999. Arqueología de la costa del lago Argentino, río Leona y pampas altas intermedias (Provincia de Santa Cruz, Argentina). Præhistoria, 3:13-33.

FRANCO, N.V. 2002. Estrategias de utilización de recursos líticos en la cuenca superior del río Santa Cruz (Argentina). Tesis doctoral inédita. Facultad de Filosofía y Letras, Universidad de Buenos Aires, Buenos Aires. MS. 2004. La organización tecnológica y el uso de escalas espaciales amplias. El caso del sur y oeste de lago Argentino. En: Temas de Arqueología. Análisis lítico, compilado por Acosta A., D. Loponte y M. Ramos, pp. 101-144. Imprenta de la Universidad Nacional de Luján, Luján.

FRANCO, N.V. y L.A. BORRERO 2003. Chorrillo Malo 2: Inicial Peopling of the Upper Santa Cruz Basin. En: Where the South Winds Blow. Ancient Evidences of Paleo South Americans, editado por L. Miotti, M. Salemme y N.
Flegenheimer, pp. 149-152. Center for the Study of the First Americans \& Texas A\&M University Press, Texas. FRANCO, N.N. CIRIGLIANO y P. AMBRÚSTOLO 2011. Semejanzas tecnológicas, diseños y prácticas funerarias al sur de la cuenca superior del río Santa Cruz: algunos ejemplos correspondientes al Holoceno Tardío. En: Bosques, montañas y cazadores: biogeografía en Patagonia Meridional, editado por Borreo L.A. y K. Borrazzo. CONICET-IMHICIHU, Buenos Aires. En prensa.

FRANCO, N, L.A. BORRERO, J. BELARDI, CARBALLO MARINA, F. MARTIN, P. CAMPAN, C. FAVIER DUBOIS, N. STADLER, M. HERNÁNDEZ LLOSAS, H. CEPEDA, A. MUÑOZ, F. BORELLA, F. MUÑOZ y I. CRUZ 1999. Arqueología del cordón Baguales y Sistema Lacustre al sur del lago Argentino (Provincia de Santa Cruz). Præhistoria, 3:65-86.

FRISON, G.C. y L.C. TODD 1986. The Colby Mammoth Site: Taphonomy and Archaeology of a Clovis Kill in Northern Wyoming. University of New Mexico Press, Albuquerque.

GARIBOTTI, I., C. PISSOLITO y R. VILLALBA 2011. Spatiotemporal pattern of primary succession in relation to mesotopographic gradients on recently deglaciated terrains in the Patagonian Andes. En: Arctic, Antarctic, and Alpine Research. En prensa.

GARCÍA, M.F., F. CARBALLO MARINA, P. CAMPAN y I. CRUZ 1999. Procesos de formación natural del registro arqueológico: lineamientos para su discusión en el lago Rico (provincia de Santa Cruz, Argentina). Præhistoria, 3:87-104.

GOÑI, R. 2010. Cambio climático y poblamiento humano durante el Holoceno tardío en Patagonia meridional. Una perspectiva arqueológica. Tesis doctoral inédita, Universidad de Buenos Aires, Buenos Aires. MS.

GOÑI, R. y G. BARRIENTOS 2000. Estudio de chenques en el lago Salitroso, Provincia de Santa Cruz. En Desde el País de los Gigantes. Perspectivas arqueológicas en Patagonia, tomo I:161-175. Universidad Nacional de la Patagonia, Río Gallegos.

GOÑI, R.G. BARRIENTOS, M.J. FIGUERERO, G.L. MENGONI, F. MENA, V. LUCERO y O. REYES 2004. Distribución espacial de entierros en la cordillera de Patagonia centro-meridional (Lago Salitroso-Paso Roballo Argentina, Entrada Baker-Río Chacabuco, Chile). Chungara, volumen especial II: 1101-1107.

GRADÍN, C. 1976. Parapetos de piedra y grabados rupestres de la meseta del lago Buenos Aires. Actas y Memorias del IV Congreso Nacional de Arqueología Argentina, tomo III (1/4):315-337. San Rafael, Mendoza. 
1971. Parapetos habitacionales en la meseta de Somuncura, Provincia de Río Negro. Relaciones de la Sociedad Argentina de Antropología, 5(2):171-185.

1962. Tres informaciones referentes a la meseta del Lago Strobel (Prov. De Santa Cruz, Argetina). Acta Praehistorica, III-IV: 144-149.

GUICHÓN, R., R. BARBERENA y L.A. BORRERO 2001. ¿Dónde y cómo aparecen los restos óseos humanos en Patagonia Austral? Anales del Instituto de la Patagonia, Serie Cs. Humanas 29:103-118.

HALLENDY, N. 2009. Tukilit: The Stone People who lived in the Wind. An Introduction to Inuksuit and Other Stone Figures of the North. Douglas \& McIntyre-University of Alaska Press, Fairbanks.

HALLER, M.J. (ed.). 2002. Geología y Recursos Naturales de Santa Cruz. Relatorio del XV Congreso Geológico Argentino, El Calafate VI-2, Buenos Aires.

INNES, J.L. 1985. Lichenometry. Progress in Physical Geography, 9:187-254.

INNES, J.L. 1986. The use of percentage cover measurements in lichenometric dating. Arctic and Alpine Research, 18:209-216.

MARTIN, F.M. 2009. Tafonomía y paleoecología de la transición Pleistoceno-Holoceno en Fuego-Patagonia. Interacción entre poblaciones humanas y carnívoros y su importancia en la formación del registro fósil. Tesis doctoral inédita, Universidad Nacional de La Plata, La Plata. MS.

MARTINIC, M. 2002. Estructuras de piedra en la Patagonia austral oriental. Anales del Instituto de la Patagonia, Serie Cs. Humanas, 30:103-115.

MARTINIC, M. y C. PORTER 2001. El hidrógrafo Phillip Parker King en aguas de Magallanes (1826-1830). Hallazgo y recuperación del testimonio de su estadía en el estrecho. Anales del Instituto de la Patagonia, Serie Cs. Humanas, 29:79-102.

MCCARROLL, D. 1994. A new approach to lichenometry: dating single-age and diachronous surfaces. The Holocene, 4:383-396.

MORENO, F.P. 1997. Viaje a la Patagonia Austral. Elefante Blanco, Buenos Aires.

PAFUNDI, L. 2006. Implicancias Arqueológicas y Modelizaciones Gráficas de los Endicamientos del Sistema Lacustre al Sur de Lago Argentino (Provincia de Santa Cruz, Argentina). Tesis de licenciatura inédita,
Facultad de Filosofía y Letras, Universidad de Buenos Aires, Buenos Aires, MS.

RAMOS, M. 1994. Propuesta terminológica para la técnica arqueológica del ensamblaje. Arqueología 3: 199-212.

REYES, O. 2002. Funebria indígena en el curso inferior del valle del río Ibáñez, margen occidental de la estepa centropatagónica (XI Región de Aisén). Anales del Instituto de la Patagonia, Serie Cs. Humanas 30:87-102.

REYES, O., C. MÉNDEZ, H. VELÁSQUEZ y V. TREJO 2006. Distribuciones espaciales y contextos arqueológicos de cazadores recolectores esteparios en Alto río Cisnes (XI Región de Aisén). Magallania, 34(2):75-90.

ROMITI, M. 2010. Estudio arqueoastronómico en el sitio Chenque 1 (Parque Nacional Lihué Calel, Provincia de La Pampa). En: Mamül Mapu. Pasado y presente desde la Arqueología pampeana, editado por Berón M., L. Luna, M. Bonomo, C. Montalvo, C. Aranda y M. Carrera Aizpitarte, pp. 143-157. Libros del Espinillo, Ayacucho. SCHIFFER, M. 1987. Formation Processes of the Archaeological Record. University of New Mexico Press, Albuquerque.

TILLEY, C., S. HAMILTON, S. HARRISON y E. ANDERSON 2000. Nature, Culture, Clitter. Distinguishing Between Cultural and Geomnorphological Landscapes; The Case of Hilltop in South-West England. Journal of Material Culture, 52(2):197-224.

WATERS, M. 1996. Principles of Geoarchaeology: a North American perspective. The University of Arizona Press, Tucson.

WEITZEL, C. 2010. El estudio de los artefactos formatizados fracturados. Contribución a la comprensión del registro arqueológico y las actividades humanas. Tesis doctoral inédita. Facultad de Filosofía y Letras, Universidad de Buenos Aires, Buenos Aires. MS.

WOOD, W.R. y D.L. JOHNSON 1978. A Survey of Disturbance processes in Archaeological Site Formation. Advances in Archaeological Method and Theory, 1:315-381.

ZILIO, L. y M. ZUBIMENDI 2011. Estructuras de entierro en la costa norte de Santa Cruz: estudio distribucional de la variabilidad espacial en el sector bahía del Oso Marino. MS.

ZUBIMENDI, M. 2010. Estrategias de uso del espacio por grupos cazadores recolectores en la costa norte de Santa Cruz y su interior inmediato. Tesis doctoral inédita, Universidad Nacional de La Plata, La Plata. MS. 